\title{
Derivation of non-local
} macroscopic traffic equations and consistent traffic pressures from microscopic car-following models

Journal Article

Author(s):

Helbing, Dirk

Publication date:

2009

Permanent link:

https://doi.org/10.3929/ethz-b-000027336

Rights / license:

In Copyright - Non-Commercial Use Permitted

Originally published in:

The European Physical Journal B 69(4), https://doi.org/10.1140/epjb/e2009-00192-5 


\title{
Derivation of non-local macroscopic traffic equations and consistent traffic pressures from microscopic car-following models
}

\author{
D. Helbing ${ }^{\mathrm{a}}$ \\ ETH Zurich, UNO D11, Universitätstr. 41, 8092 Zurich, Switzerland
}

Received 19 May 2008 / Received in final form 9 October 2008

Published online 3 June 2009 - (c) EDP Sciences, Società Italiana di Fisica, Springer-Verlag 2009

\begin{abstract}
This contribution compares several different approaches allowing one to derive macroscopic traffic equation directly from microscopic car-following models. While it is shown that some conventional approaches lead to theoretical problems, it is proposed to use an approach reminding of smoothed particle hydrodynamics to avoid gradient expansions. The derivation circumvents approximations and, therefore, demonstrates the large range of validity of macroscopic traffic equations, without the need of averaging over many vehicles. It also gives an expression for the "traffic pressure", which generalizes previously used formulas. Furthermore, the method avoids theoretical inconsistencies of macroscopic traffic models, which have been criticized in the past by Daganzo and others.
\end{abstract}

PACS. 89.40.Bb Land transportation - 45.70.Vn Granular models of complex systems; traffic flow 47.10.ab Conservation laws and constitutive relations

\section{Introduction}

In order to describe the dynamics of traffic flows, a large number of mathematical models has been developed. The analysis of the spatio-temporal features and statistics of traffic patterns has often been done with methods from non-linear dynamics and statistical physics. An overview of modeling approaches and methods is, for example, given in references [1-4]. Among these are cellular automata, "microscopic" car-following models, "mesoscopic" gas-kinetic, and macroscopic traffic models.

Cellular automata can often be interpreted as discretized versions of car-following models, while gas-kinetic models have frequently been used to derive macroscopic from microscopic models. Such derivations were driven by the desire to improve phenomenological specifications of macroscopic traffic models [5-7], which were criticized to have unrealistic properties [21]. However, the derivation of gas-kinetic models from car-following models usually simplifies the interactions among vehicles by a collisional approach assuming immediate braking maneuvers. Moreover, the derivation of macroscopic traffic models from gas-kinetic ones terminates an infinite and poorly converging series expansion, which replaces dynamical equations for higher moments of the velocity distribution by simplified equilibrium relationships [8].

Although this leads to macroscopic equations which work well in most theoretical and practical aspects [9], the implications of the approximations are hardly known.

\footnotetext{
a e-mail: dhelbing@ethz.ch
}

Moreover, the approach seems to require an averaging over at least 100 vehicles for each speed class and spatial location. While this constitutes no problem for gases with $10^{23}$ particles within a small volume, for traffic flows this would require an averaging over spatial intervals much greater than the scale on which traffic flow changes. Hence, it is not well understood, whether or why macroscopic traffic equations can be used at all.

In this paper, we will therefore focus on attempts to derive macroscopic traffic equations directly from microscopic ones. Doing so, we will compare three different approaches: first, we study the gradient expansion approach in Section 2. Second, we turn to the linear interpolation approach in Section 3. Third, we discuss an approach reminding of smoothed particle hydrodynamics in Section 4 and compare the results with macroscopic traffic models such as the Payne model, the Aw-Rascle model, and a non-local traffic model. In the conclusions of Section 5, we summarize and discuss our results, in particular with regard to the mathematical form of the traffic pressure and the theoretical consistency of macroscopic traffic models.

\section{The gradient expansion approach}

Already in the 1970's, Payne [10,11] used a gradient expansion approach to derive a macroscopic velocity equation complementing the continuity equation

$$
\frac{\partial \rho}{\partial t}+\frac{\partial}{\partial x}[\rho(x, t) V(x, t)]=0
$$


It relates the vehicle density $\rho(x, t)$ at location $x$ and time $t$ with the average velocity $V(x, t)$ or the vehicle flow

$$
Q(x, t)=\rho(x, t) V(x, t),
$$

respectively, and describes the conservation of the number of vehicles [12].

Payne derived his model from Newell's car-following model [13]

$$
v_{i}(t+\tau)=v_{\mathrm{o}}\left(d_{i}(t)\right),
$$

which assumes that the speed $v_{i}(t)$ of vehicle $i$ at time $t$ will be adjusted with a delay of $\tau$ to some optimal speed $v_{\mathrm{o}}$, which depends on the distance $d_{i}(t)=x_{i-1}(t)-$ $x_{i}(t)$ between the location of the leading vehicle $x_{i-1}(t)$ and the location $x_{i}(t)$ of the following car.

Payne identified microscopic and macroscopic velocities as follows:

$$
\begin{aligned}
v_{i}(t+\tau) & =V(x+V \tau, t+\tau) \\
& \approx V(x, t)+V \tau \frac{\partial V(x, t)}{\partial x}+\tau \frac{\partial V(x, t)}{\partial t} .
\end{aligned}
$$

Then, Taylor approximations (gradient expansions) were used in several places. For example, Payne substituted the inverse of the distance $d_{i}$ to the leading vehicle by the density $\rho$ at the place $x+d_{i}(t) / 2$ in the middle between the leading and the following vehicle. In this way, he obtained

$$
\begin{aligned}
\frac{1}{d_{i}(t)} & =\rho\left(x+\frac{d_{i}(t)}{2}, t\right)=\rho\left(x+\frac{1}{2 \rho}, t\right) \\
& \approx \rho(x, t)+\frac{1}{2 \rho} \frac{\partial \rho(x, t)}{\partial x} .
\end{aligned}
$$

When defining the so-called equilibrium velocity $V_{\mathrm{e}}(\rho)$ through

$$
V_{\mathrm{e}}(\rho)=v_{\mathrm{o}}\left(\frac{1}{\rho}\right) \quad \text { or } \quad V_{\mathrm{e}}\left(\frac{1}{d_{i}}\right)=v_{\mathrm{o}}\left(d_{i}\right)
$$

a first order Taylor approximation and equation (5) imply

$$
\begin{aligned}
v_{\mathrm{o}}\left(d_{i}(t)\right) & =V_{\mathrm{e}}\left(\frac{1}{d_{i}(t)}\right) \\
& \approx V_{\mathrm{e}}(\rho(x, t))+\frac{1}{2 \rho(x, t)} \frac{d V_{\mathrm{e}}(\rho)}{d \rho} \frac{\partial \rho(x, t)}{\partial x} .
\end{aligned}
$$

Starting from the previous equations, one finally arrives at Payne's macroscopic velocity equation

$$
\frac{\partial V}{\partial t}+V \frac{\partial V}{\partial x}=\frac{1}{\tau}\left[V_{\mathrm{e}}(\rho)-\frac{D(\rho)}{\rho} \frac{\partial \rho}{\partial x}-V(x, t)\right],
$$

where we have introduced the density-dependent diffusion

$$
D(\rho)=-\frac{1}{2} \frac{d V_{\mathrm{e}}(\rho)}{\partial \rho}=\frac{1}{2}\left|\frac{d V_{\mathrm{e}}(\rho)}{d \rho}\right| \geq 0 .
$$

The single terms of equation (8) have the following interpretation: the term $V \partial V / \partial x$ is called the transport term and describes a motion of the velocity profile with the vehicles. The term $-[D(\rho) /(\rho \Delta t)] \partial \rho / \partial x$ is called anticipation term, as it reflects the reaction of drivers to the traffic situation in front of them. The relaxation term $\left[V_{\mathrm{e}}(\rho)-V\right] / \Delta t$ delineates the adaptation of the average velocity $V(x, t)$ to the density-dependent equilibrium velocity $V_{\mathrm{e}}(\rho)$ with a delay $\tau$.

Other authors have applied similar gradient expansions to the optimal velocity model defined by

$$
\frac{d v_{i}(t)}{d t}=\frac{1}{\tau}\left[v_{\mathrm{o}}\left(d_{i}(t)\right)-v_{i}(t)\right]
$$

with $d d_{i} / d t=v_{i-1}(t)-v_{i}(t)$, see e.g. references $[15,16]$. Equation (10) results from the Newell model (3) by a firstorder Taylor approximation $v_{i}(t+\tau) \approx v_{i}(t)+\tau d v_{i} / d t$. Regarding the derivation of macroscopic traffic equations from the optimal velocity model, it is also worth reading references $[15,16]$.

One weakness of the gradient expansion approach is that its validity implicitly requires small gradients. However, it is well-known that many microscopic and macroscopic traffic equations give rise to emergent traffic jams, which are related with steep gradients. That calls for the consideration of higher-order terms and leads to macroscopic traffic equations that are not anymore simple and well tractable (even numerically). Let us, therefore, study other approaches to determine macroscopic from microscopic equations.

\section{The linear interpolation approach}

The optimal velocity model may be also written in the form

$$
\frac{d v_{i}}{d t}=a_{i}(t)=\frac{v^{0}-v_{i}(t)}{\tau}+f\left(d_{i}(t)\right),
$$

where $a_{i}(t)$ denotes the acceleration, $v^{0}$ the "desired velocity" or "free speed", and

$$
f\left(d_{i}\right)=\frac{v_{\mathrm{o}}\left(d_{i}\right)-v^{0}}{\tau} \leq 0
$$

the repulsive interaction among the leading vehicle $i-1$ and its follower $i$.

In reference [17], it has been suggested to establish a micro-macro link between microscopic and macroscopic traffic variables by the definitions

$$
\begin{aligned}
\rho(x, t)= & \frac{\frac{1}{x_{i}(t)-x_{i+1}(t)}\left[x_{i-1}(t)-x\right]}{x_{i-1}(t)-x_{i}(t)} \\
& +\frac{\frac{1}{x_{i-1}(t)-x_{i}(t)}\left[x-x_{i}(t)\right]}{x_{i-1}(t)-x_{i}(t)}, \\
V(x, t)= & \frac{v_{i}(t)\left[x_{i-1}(t)-x\right]+v_{i-1}(t)\left[x-x_{i}(t)\right]}{x_{i-1}(t)-x_{i}(t)}, \\
A(x, t)= & \frac{a_{i}(t)\left[x_{i-1}(t)-x\right]+a_{i-1}(t)\left[x-x_{i}(t)\right]}{x_{i-1}(t)-x_{i}(t)} .
\end{aligned}
$$


These definitions assume that the macroscopic variables in the vehicle locations $x=x_{i}(t)$ would be given by the microscopic ones, while in locations $x$ between two vehicles, they would be defined by linear interpolation.

Let us consider the consequences of such an approach. For this, we determine the partial derivative of

$$
G(x, t)=\frac{g_{i}(t)\left[x_{i-1}(t)-x\right]+g_{i-1}(t)\left[x-x_{i}(t)\right]}{x_{i-1}(t)-x_{i}(t)}
$$

with respect to $x$, which gives

$$
\frac{\partial G(x, t)}{\partial x}=\frac{-g_{i}(t)+g_{i-1}(t)}{x_{i-1}(t)-x_{i}(t)}
$$

for any specification of $g_{i}(t)$, for example, $g_{i}(t)=v_{i}(t)$. The partial derivative with respect to time is

$$
\begin{aligned}
\frac{\partial G(x, t)}{\partial t}= & \frac{\frac{d g_{i}(t)}{d t}\left[x_{i-1}(t)-x\right]+g_{i}(t) \frac{d x_{i-1}(t)}{d t}}{x_{i-1}(t)-x_{i}(t)} \\
& +\frac{\frac{d g_{i-1}(t)}{d t}\left[x-x_{i}(t)\right]-g_{i-1}(t) \frac{d x_{i}(t)}{d t}}{x_{i-1}(t)-x_{i}(t)} \\
& -\frac{\left(\frac{d x_{i-1}(t)}{d t}-\frac{d x_{i}(t)}{d t}\right) g_{i}(t)\left[x_{i-1}(t)-x\right]}{\left[x_{i-1}(t)-x_{i}(t)\right]^{2}} \\
& -\frac{\left(\frac{d x_{i-1}(t)}{d t}-\frac{d x_{i}(t)}{d t}\right) g_{i-1}(t)\left[x-x_{i}(t)\right]}{\left[x_{i-1}(t)-x_{i}(t)\right]^{2}}
\end{aligned}
$$

For $g_{i}(t)=v_{i}(t)=d x_{i} / d t$ and with $d v_{i} / d t=a_{i}(t)$, this formula simplifies to the following expression:

$$
\begin{aligned}
\frac{\partial V(x, t)}{\partial t}= & \frac{a_{i}(t)\left[x_{i-1}(t)-x\right]+v_{i}(t) v_{i-1}(t)}{x_{i-1}(t)-x_{i}(t)} \\
& +\frac{a_{i-1}(t)\left[x-x_{i}(t)\right]-v_{i-1}(t) v_{i}(t)}{x_{i-1}(t)-x_{i}(t)} \\
& -\frac{v_{i-1}(t)-v_{i}(t)}{x_{i-1}(t)-x_{i}(t)} \\
& \times \frac{v_{i}(t)\left[x_{i-1}(t)-x\right]+v_{i-1}(t)\left[x-x_{i}(t)\right]}{x_{i-1}(t)-x_{i}(t)} \\
= & A(x, t)-\frac{\partial V(x, t)}{\partial x} V(x, t) .
\end{aligned}
$$

As a consequence, we find the exact relationship

$$
\frac{\partial V(x, t)}{\partial t}+V(x, t) \frac{\partial V(x, t)}{\partial x}=A(x, t) .
$$

This would be fully compatible with Payne's macroscopic traffic equation (8), if

$$
A(x, t)=\frac{1}{\tau}\left[V_{\mathrm{e}}(\rho)-V(x, t)\right]-\frac{D(\rho)}{\tau \rho(x, t)} \frac{\partial \rho}{\partial x} .
$$

However, the expression for $g_{i}(t)=1 /\left[x_{i-1}(t)-x_{i}(t)\right]$ does not simplify in a way that would finally lead to the continuity equation (1). Therefore, a micro-macro link based on the linear interpolation (16) of the microscopic variables $g_{i}(t)$ does not exactly imply the conservation of the number of vehicles, i.e. it is theoretically not consistent. Nevertheless, it works surprisingly well in practise [17].

In the next section, we will see that the interpolation approach fails because it does not reflect the nonlocality of the correct macroscopic traffic equations, see equation (39) or (47). The dependence on gradients makes the model too isotropic, while vehicles should only respond to the traffic situation ahead of them, but not behind them. This problem is usually taken care of by hyperbolic schemes such as the Godunov scheme, as used for example in reference [18]. This scheme naturally discretizes the velocity in a downwind way, which avoids the isotropy problem of Payne's model and similar ones [21].

To avoid this problem, reference [19] suggests a hybrid Lagrangian approach. This is based on a transformation into Lagrangian coordinates, i.e. a moving coordinate system. As a result, the continuity equation (1) becomes linear. For piecewise linear $\rho$ and $V$, the result can then be transformed back into Eulerian coordinates, i.e. into the stationary frame of reference. In the following, we will present an alternative method that yields macroscopic traffic equations from microscopic ones directly, without the need of transformation into Lagrangian coordinates.

\section{An approach reminding of smooth particle hydrodynamics}

\subsection{Derivation of the continuity equation}

In this section, we will start with the derivation of the continuity equation from the equation of motion $d x_{i} / d t=v_{i}$, using a "trick" that I learned from Isaac Goldhirsch. For this, we represent the location $x_{i}(t)$ of an element $i$ in space by a delta function $\delta\left(x-x_{i}(t)\right)$, which may be treated here like a very narrow Gaussian distribution. Moreover, we introduce a symmetrical smoothing function

$$
s\left(x^{\prime}-x\right)=s\left(\left|x^{\prime}-x\right|\right)=s\left(x-x^{\prime}\right),
$$

for example, a Gaussian distribution with a finite variance or a differentiable approximation of a triangular function or a rectangular one. The smoothing function shall be normalized by demanding

$$
\int_{-\infty}^{\infty} d x^{\prime} s\left(x^{\prime}-x\right)=1
$$

for any value of $x$. With this, we define the local density

$$
\begin{aligned}
\rho(x, t) & =\int_{-\infty}^{\infty} d x^{\prime} s\left(x^{\prime}-x\right) \sum_{i} \delta\left(x^{\prime}-x_{i}(t)\right) \\
& =\sum_{i} s\left(x_{i}(t)-x\right) .
\end{aligned}
$$


Herein, we sum up over all particles $i$. Note that the replacement of the conventional formula $\sum_{i} \delta\left(x_{i}(t)-x\right)$ for the vehicle density by the formula $\sum_{i} s\left(x_{i}(t)-x\right)$ reminds of a substitution of point-like particles by "fuzzy" particles, which is the idea behind smoothed particle hydrodynamics. Nevertheless it should be remembered that we have formally related the smoothing function $s\left(x^{\prime}-x\right)$ to locations $x^{\prime}$ in the stationary frame of reference, and not to the moving vehicles themselves.

Now, we define the average velocity $V(x, t)$ as usual via a weighted average with the weight function $\delta\left(x^{\prime}-\right.$ $\left.x_{i}(t)\right) s\left(x^{\prime}-x\right)$ :

$$
\begin{aligned}
V(x, t) & =\frac{\int_{-\infty}^{\infty} d x^{\prime} \sum_{i} v_{i}(t) \delta\left(x^{\prime}-x_{i}(t)\right) s\left(x^{\prime}-x\right)}{\int_{-\infty}^{\infty} d x^{\prime} \sum_{i} \delta\left(x-x_{i}(t)\right) s\left(x^{\prime}-x\right)} \\
= & \frac{\int_{-\infty}^{\infty} d x^{\prime} \sum_{i} v_{i}(t) \delta\left(x^{\prime}-x_{i}(t)\right) s\left(x^{\prime}-x\right)}{\rho(x, t)} \\
= & \frac{\sum_{i} v_{i}(t) s\left(x_{i}(t)-x\right)}{\sum_{i} s\left(x_{i}(t)-x\right)} \\
= & \frac{\sum_{i} v_{i}(t) s\left(x_{i}(t)-x\right)}{\rho(x, t)} .
\end{aligned}
$$

This implies the well-known fluid-dynamic flow relationship

$$
Q(x, t)=\rho(x, t) V(x, t) .
$$

Differentiation of equation (24) with respect to time and application of the chain rule gives

$$
\begin{aligned}
& \frac{\partial \rho(x, t)}{\partial t}= \\
& \quad \int_{-\infty}^{\infty} d x^{\prime} \sum_{i}\left(-\frac{d x_{i}}{d t}\right) \cdot\left[\frac{\partial}{\partial x^{\prime}} \delta\left(x^{\prime}-x_{i}(t)\right)\right] s\left(x^{\prime}-x\right) \\
& \quad \int_{-\infty}^{\infty} d x^{\prime} \sum_{i} v_{i}(t) \delta\left(x^{\prime}-x_{i}(t)\right)\left[\frac{\partial}{\partial x^{\prime}} s\left(x^{\prime}-x\right)\right],
\end{aligned}
$$

where we have applied partial integration to obtain the last results. That is, we have used the theorem

$$
\begin{aligned}
\int_{-\infty}^{\infty} d x^{\prime}\left[\frac{\partial}{\partial x^{\prime}} u\left(x^{\prime}\right)\right] v\left(x^{\prime}\right)= & {[u(x) v(x)]_{-\infty}^{\infty} } \\
& -\int_{-\infty}^{\infty} u\left(x^{\prime}\right)\left[\frac{\partial}{\partial x^{\prime}} v\left(x^{\prime}\right)\right],
\end{aligned}
$$

considering the vanishing of the first term after the equality sign due to the vanishing of $u(x) v(x)$ at the bound- aries. Taking into account the symmetry of the smoothing function $s\left(x^{\prime}-x\right)$, we may replace $\partial s\left(x^{\prime}-x\right) / \partial x^{\prime}$ by $-\partial s\left(x^{\prime}-x\right) / \partial x$, which finally yields equation (1) as follows:

$$
\begin{aligned}
\frac{\partial \rho(x, t)}{\partial t} & =-\frac{\partial}{\partial x} \int_{-\infty}^{\infty} d x^{\prime} \sum_{i} v_{i}(t) \delta\left(x^{\prime}-x_{i}(t)\right) s\left(x^{\prime}-x\right) \\
& =-\frac{\partial}{\partial x}[\rho(x, t) V(x, t)]
\end{aligned}
$$

To obtain this desired result, we have finally applied the definition $(26)$ of the average velocity $V(x, t)$. As a consequence of this, the validity of the continuity equation does not require an averaging over large numbers of entities, i.e. macroscopic volumes to average over. This makes the equation absolutely fundamental and explains its large range of validity.

\subsection{Derivation of the macroscopic velocity equation}

In order to derive the equation for the average velocity, we start by deriving the formula

$$
\rho(x, t) V(x, t)=\sum_{i} v_{i}(t) s\left(x_{i}(t)-x\right)
$$

for the vehicle flow with respect to time. This gives

$$
\begin{aligned}
\frac{\partial}{\partial t}[\rho(x, t) V(x, t)]= & \sum_{i} \frac{d v_{i}(t)}{d t} s\left(x_{i}(t)-x\right) \\
& +\sum_{i} v_{i}(t) \frac{\partial}{\partial x_{i}}\left[s\left(x_{i}(t)-x\right)\right] \frac{d x_{i}(t)}{d t} \\
= & \sum_{i} a_{i}(t) s\left(x_{i}(t)-x\right) \\
& -\frac{\partial}{\partial x} \sum_{i}\left[v_{i}(t)\right]^{2} s\left(x_{i}(t)-x\right) .
\end{aligned}
$$

Introducing $\delta v_{i}(x, t)=v_{i}(t)-V(x, t)$ and defining the velocity variance

$$
\begin{aligned}
\theta(x, t) & =\frac{\int_{-\infty}^{\infty} d x^{\prime} \sum_{i}\left[v_{i}(t)-V(x, t)\right]^{2} \delta\left(x^{\prime}-x_{i}(t)\right) s\left(x^{\prime}-x\right)}{\int_{-\infty}^{\infty} d x^{\prime} \sum_{i} \delta\left(x^{\prime}-x_{i}(t)\right) s\left(x^{\prime}-x\right)} \\
& =\frac{\sum_{i}\left[v_{i}(t)-V(x, t)\right]^{2} s\left(x_{i}(t)-x\right)}{\sum_{i} s\left(x_{i}(t)-x\right)} \\
& =\frac{\sum_{i}\left[\delta v_{i}(x, t)\right]^{2} s\left(x_{i}(t)-x\right)}{\rho(x, t)}
\end{aligned}
$$


similarly to the average velocity (26), we can make the decomposition

$$
\begin{gathered}
\sum_{i}\left[v_{i}(t)\right]^{2} s\left(x_{i}(t)-x\right)= \\
\sum_{i}\left[V(x, t)+\delta v_{i}(x, t)\right]^{2} s\left(x_{i}(t)-x\right) \\
=\sum_{i}\left\{[V(x, t)]^{2}+2 V(x, t) \delta v_{i}(x, t)\right. \\
\left.+\left[\delta v_{i}(x, t)\right]^{2}\right\} s\left(x_{i}(t)-x\right) \\
=\rho(x, t)[V(x, t)]^{2}+2 \rho(x, t) V(x, t)[V(x, t)-V(x, t)] \\
\quad+\rho(x, t) \theta(x, t), \quad
\end{gathered}
$$

where we have considered

$$
\begin{aligned}
\sum_{i} \delta v_{i}(x, t) s\left(x_{i}(t)-x\right) & =\sum_{i}\left[v_{i}(t)-V(x, t)\right] s\left(x_{i}(t)-x\right) \\
& =Q(x, t)-\rho(x, t) V(x, t)=0,(35)
\end{aligned}
$$

see equations (26) and (25). Altogether, we get

$$
\begin{aligned}
\frac{\partial}{\partial t}[\rho(x, t) V(x, t)]= & -\frac{\partial}{\partial x}\left\{\rho(x, t)\left[V(x, t)^{2}+\theta(x, t)\right]\right\} \\
& +\sum_{i} a_{i}(t) s\left(x_{i}(t)-x\right)
\end{aligned}
$$

Now, we carry out the partial differentiation applying the product rule of Calculus. Taking into account

$$
\rho(x, t) \frac{\partial V(x, t)}{\partial t}=-V(x, t) \frac{\partial \rho(x, t)}{\partial t}+\frac{\partial}{\partial t}[\rho(x, t) V(x, t)]
$$

and

$$
\begin{aligned}
& \frac{\partial}{\partial x}\{[\rho(x, t) V(x, t)] V(x, t)\}=\rho(x, t) V(x, t) \frac{\partial V}{\partial x} \\
&+V(x, t) \frac{\partial}{\partial x}[\rho(x, t) V(x, t)],
\end{aligned}
$$

with equation (36) we obtain the following:

$$
\begin{aligned}
\rho(x, t) \frac{\partial V(x, t)}{\partial t}= & -V(x, t) \frac{\partial \rho(x, t)}{\partial t} \\
& -V(x, t) \frac{\partial}{\partial x}[\rho(x, t) V(x, t)] \\
& -\rho(x, t) V(x, t) \frac{\partial V(x, t)}{\partial x} \\
& -\frac{\partial}{\partial x}[\rho(x, t) \theta(x, t)] \\
& +\sum_{i} a_{i}(t) s\left(x_{i}(t)-x\right) .
\end{aligned}
$$

Inserting the continuity equation (30) for $\partial \rho / \partial t$ and dividing the above equation by $\rho(x, t)$ finally yields the velocity equation

$$
\begin{gathered}
\frac{\partial V(x, t)}{\partial t}+V(x, t) \frac{\partial V(x, t)}{\partial x}=-\frac{1}{\rho(x, t)} \frac{\partial}{\partial x}[\rho(x, t) \theta(x, t)] \\
+\frac{1}{\rho(x, t)} \sum_{i} a_{i}(t) s\left(x_{i}(t)-x\right)
\end{gathered}
$$

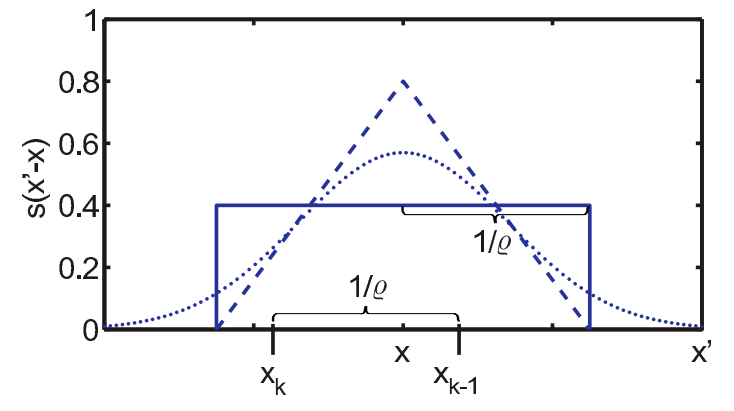

Fig. 1. Illustration of rectangular $(-)$, triangular (--), and Gaussian $(\cdots)$ smoothing functions $s\left(x^{\prime}-x\right) . x_{k}$ and $x_{k-1}$ are the locations of the two closest vehicles $k$ and $k-1$ with respect to a reference location $x$. Their distance $1 / \varrho=x_{k-1}-$ $x_{k}$ determines the size $2 / \varrho$ of the smoothing range chosen in the calculations of the main text.

Inserting equation (11) for $a_{i}(t)$, we find

$$
\begin{aligned}
& \sum_{i} a_{i}(t) s\left(x_{i}(t)-x\right)= \\
& \sum_{i}\left[\frac{v^{0}-v_{i}}{\tau}+\sum_{i} f\left(d_{i}(t)\right)\right] s\left(x_{i}(t)-x\right) \\
&=\frac{v^{0}-V(x, t)}{\tau}+\sum_{i} f\left(d_{i}(t)\right) s\left(x_{i}(t)-x\right) .
\end{aligned}
$$

For further simplification, let us now specify the smoothing function by the rectangular function

$$
s\left(x_{i}-x\right)=\frac{\varrho}{2} \cdot\left\{\begin{array}{l}
1 \text { if }\left|x_{i}-x\right| \leq 1 / \varrho \\
0 \text { otherwise }
\end{array}\right.
$$

with a large enough smoothing window of length $\Delta x=$ $2 / \varrho$ (see Fig. 1). Then, the number of vehicles $i$ within the smoothing interval $[x-1 / \varrho, x+1 / \varrho]$ is expected to be $\rho \Delta x=2 \rho / \varrho$, where $\rho$ represents the average vehicle density in this interval. Therefore,

$$
\rho(x, t)=\sum_{i} s\left(x_{i}(t)-x\right)=\frac{2 \rho}{\varrho} \frac{\varrho}{2}=\rho,
$$

which shows the consistency of this approach.

If the smoothing parameter $\varrho$ is specified via the inverse vehicle distance

$$
\varrho=\varrho_{k}=\frac{1}{d_{k}}=\frac{1}{x_{k-1}-x_{k}}=\rho(x, t) \text { for } x_{k}<x \leq x_{k-1},
$$

the smoothing window of length $\Delta x=2 / \varrho$ will usually contain only two vehicles $k-1$ and $k$ with $x_{k}<x \leq x_{k-1}$ (see Fig. 1). With this, the sum over $i$ reduces to two terms with $i=k$ and $i=k-1$ only. This finally yields

$$
\begin{aligned}
\rho(x, t) V(x, t) & =\sum_{i} v_{i}(t) s\left(x_{i}(t)-x\right) \\
& =v_{k}(t) s\left(x_{k}(t)-x\right)+v_{k-1}(t) s\left(x_{k-1}(t)-x\right) \\
& =\frac{\varrho}{2}\left[v_{k-1}(t)+v_{k}(t)\right] \\
& =\rho(x, t) \frac{v_{k-1}(t)+v_{k}(t)}{2}
\end{aligned}
$$


and, considering equation (44),

$$
\begin{aligned}
\sum_{i} s\left(x_{i}(t)-x\right) f\left(d_{i}(t)\right)= & \frac{\varrho}{2} f\left(d_{k}\right)+\frac{\varrho}{2} f\left(d_{k-1}\right) \\
= & \frac{\varrho}{2} f\left(\frac{1}{\varrho_{k}}\right)+\frac{\varrho}{2} f\left(\frac{1}{\varrho_{k-1}}\right) \\
= & \frac{\rho(x, t)}{2} f\left(\frac{1}{\rho(x, t)}\right) \\
& +\frac{\rho(x, t)}{2} f\left(\frac{1}{\rho(x+1 / \rho, t)}\right) .
\end{aligned}
$$

In summary, the macroscopic velocity equation related to the optimal velocity model corresponds to ${ }^{1}$

$$
\begin{aligned}
\frac{\partial V(x, t)}{\partial t}+ & V(x, t) \frac{\partial V(x, t)}{\partial x}= \\
- & \frac{1}{\rho(x, t)} \frac{\partial}{\partial x}[\rho(x, t) \theta(x, t)]+\frac{v^{0}-V(x, t)}{\tau} \\
& +\frac{1}{2} f\left(\frac{1}{\rho(x, t)}\right)+\frac{1}{2} f\left(\frac{1}{\rho(x+1 / \rho, t)}\right) .
\end{aligned}
$$

Note that the last line of this equation contains more terms, if more than 2 vehicles are located in the spatial interval between $x-1 / \varrho$ and $x+1 / \varrho$, as it can happen due to density variations. Since this does not affect a numerical implementation of the macroscopic equations (40) and (41), we do not need to be concerned about this. Equation (43) anyway remains unchanged.

At the cost of less straight-forward analytical evaluation, it is also possible to use other than rectangular smoothing functions (see Fig. 1). A triangular function, for example, puts less weight on the boundaries of the smoothing window, so it will make little difference whether there are 2 or 3 cars in the smoothing range. Using the specification

$$
s\left(x_{i}-x\right)=\max \left[\varrho\left(1-\varrho\left|x_{i}-x\right|\right), 0\right]
$$

and considering

$\left|x_{k-1}-x\right|+\left|x-x_{k}\right|=\left(x_{k-1}-x\right)+\left(x-x_{k}\right)=x_{k-1}-x_{k}=\frac{1}{\varrho}$

shows that a triangular specification leads to the same consistent density measurement:

$$
\begin{aligned}
\rho(x, t) & =s\left(x_{k-1}(t)-x\right)+s\left(x_{k}(t)-x\right) \\
& =2 \varrho-\varrho^{2}\left(x_{k-1}-x_{k}\right)=\varrho .
\end{aligned}
$$

\subsection{Discussion of the non-locality}

The crucial point of equation (47) is its non-locality. The dependence on $x+1 / \rho(x, t)$ reflects the anticipatory behavior of drivers, who react to the traffic situation ahead

\footnotetext{
1 If another smoothing function is applied, the last term of equation (47) is replaced by a similar weighted mean value, as equation (41) reveals, but the essence stays the same. That is, the way of looking at the microscopic equations (i.e. the way of defining the density and velocity moments) potentially has some influence on the dynamics, but it is expected to be small.
}

of them. From the point of view of traffic simulation, the non-locality does not constitute a problem. Non-local traffic models such as the gas-kinetic based traffic model summarized in Appendix A can be even more efficient numerically than second-order models with diffusion terms, that would result from a gradient expansion.

In fact, the reason for the numerical inefficiency of explicit solvers for partial differential equations is the diffusion instability, which must be avoided by small time discretizations [20]. As pointed out by Daganzo [21], a diffusion term also implies theoretical inconsistencies such as the possible occurence of negative velocities at upstream jam fronts. Therefore, it should be underlined that numerical inefficiencies and theoretical inconsistencies can be avoided by working with the non-local velocity equation rather than with the gradient expansion of it, which will be looked at in the next section.

\subsection{Comparison with other macroscopic traffic models}

\subsubsection{The Payne model}

Despite the before-mentioned problems, we will now carry out a Taylor expansion of the non-local terms in equation (47), exclusively for the sake of comparison with other traffic models. A first-order approximation gives

$$
\begin{gathered}
f\left(\frac{1}{\rho(x+1 / \rho, t)}\right) \approx f\left(\frac{1}{\rho(x, t)+\frac{\partial \rho(x, t)}{\partial x} \frac{1}{\rho(x, t)}}\right) \\
\approx f\left(\frac{1}{\rho(x, t)}\left(1-\frac{\partial \rho(x, t)}{\partial x} \frac{1}{\rho(x, t)^{2}}\right)\right) \\
\approx f\left(\frac{1}{\rho(x, t)}\right)+\frac{d f(d)}{d d} \cdot\left(-\frac{\partial \rho(x, t)}{\partial x} \frac{1}{\rho(x, t)^{3}}\right)
\end{gathered}
$$

where we have applied the geometric series expansion $1 /(1-z) \approx 1+z+\ldots$ Note that the relation $\rho=1 / d$ and

$$
V_{\mathrm{e}}(\rho)=V_{\mathrm{e}}\left(\frac{1}{d}\right)=v_{\mathrm{o}}(d)=v^{0}+\tau f(d)=v^{0}+\tau f\left(\frac{1}{\rho}\right)
$$

imply

$$
\begin{aligned}
\frac{d f(d)}{d d} & =\left(\frac{d}{d \rho} \frac{V_{\mathrm{e}}(\rho)-v^{0}}{\tau}\right) \frac{d \rho}{d d}=\frac{1}{\tau} \frac{d V_{\mathrm{e}}(\rho)}{d \rho} \cdot\left(-\frac{1}{d^{2}}\right) \\
& =-\frac{\rho^{2}}{\tau} \frac{d V_{\mathrm{e}}(\rho)}{d \rho}
\end{aligned}
$$

Therefore, using equation (46), we finally obtain:

$\sum_{i} s\left(x_{i}(t)-x\right) f(t) \approx \rho(x, t) f\left(\frac{1}{\rho(x, t)}\right)+\frac{1}{2 \tau} \frac{d V_{\mathrm{e}}(\rho)}{d \rho} \frac{\partial \rho(x, t)}{\partial x}$.

Considering $V_{\mathrm{e}}(\rho)=v^{0}+\tau f(\rho)$ and defining the "traffic pressure" as

$$
P(x, t)=\rho(x, t) \theta(x, t)+\frac{v^{0}-V_{\mathrm{e}}(\rho)}{2 \tau},
$$


the corresponding macroscopic velocity equation becomes

$$
\begin{aligned}
\frac{\partial V(x, t)}{\partial t}+V(x, t) \frac{\partial V(x, t)}{\partial x}= & -\frac{1}{\rho(x, t)} \frac{\partial P(x, t)}{\partial x} \\
& +\frac{V_{\mathrm{e}}(\rho)-V(x, t)}{\tau} .
\end{aligned}
$$

If the velocity variance $\theta$ is zero, this model corresponds exactly to Payne's macroscopic traffic model with the pressure term $[10,11]$

$$
P(\rho)=\frac{V^{0}-V_{\mathrm{e}}(\rho)}{2 \tau}
$$

As a check of consistency between the Payne model and the optimal velocity model, one may perform an instability analysis of both models. Such an analysis is carried out in reference [22] and demonstrates indeed that the instability conditions and the characteristic velocities are compatible, as expected.

\subsubsection{The macroscopic traffic model by Aw and Rascle}

Note that Daganzo has seriously criticized macroscopic traffic equations of the type (56) [23]. For example, he studied the case of a vehicle queue of maximum density $\rho=\rho_{\text {jam }}$ and speed $V=V_{\mathrm{e}}\left(\rho_{\text {jam }}\right)=0$, the end of which was assumed to be at some location $x=x_{0}$. In this situation, equation (56) predicts $V=0$ and $d V / d t=\partial V / \partial t+V \partial V / \partial x<0$ for the last vehicle in the queue, i.e. the occurence of negative velocities, if pressure relations such as $P=\rho \theta_{0}-\eta_{0} \partial V / \partial x$ with non-negative parameters $\theta_{0}$ and $\eta_{0}$ are assumed [7].

In order to overcome Daganzo's criticism, Aw and Rascle have proposed the macroscopic velocity equation

$$
\frac{\partial}{\partial t}[V+p(\rho)]+V \frac{\partial}{\partial x}[V+p(\rho)]=0
$$

with $p(\rho)=\rho^{\gamma}[23]$. Let us study, how this model relates to the previous macroscopic models. For this purpose, let us apply the chain rule of Calculus to obtain

$$
\begin{aligned}
\frac{\partial V(x, t)}{\partial t}+ & V(x, t) \frac{\partial V(x, t)}{\partial x}= \\
& -\frac{d p(\rho)}{d \rho} \frac{\partial \rho(x, t)}{\partial t}-V(x, t) \frac{d p(\rho)}{d \rho} \frac{\partial \rho(x, t)}{\partial x} .
\end{aligned}
$$

Inserting the continuity equation (30) for $\partial \rho / \partial t$ on the right-hand side, we get

$$
\begin{aligned}
\frac{\partial V(x, t)}{\partial t}+V(x, t) \frac{\partial V(x, t)}{\partial x}= & \frac{d p(\rho)}{d \rho} \frac{\partial}{\partial x}[\rho(x, t) V(x, t)] \\
& -V(x, t) \frac{d p(\rho)}{d \rho} \frac{\partial \rho(x, t)}{\partial x} \\
= & \rho(x, t) \frac{d p(\rho)}{d \rho} \frac{\partial V(x, t)}{\partial x}
\end{aligned}
$$

This model can be rigorously derived from particular carfollowing models [18]. By comparison with the macroscopic velocity equation (56) we see that the model by
Aw and Rascle does not have a relaxation term $\left[V_{\mathrm{e}}(\rho)-\right.$ $V(x, t)] / \tau$, which would correspond to the limit $\tau \rightarrow \infty$. Moreover, we find

$$
-\frac{1}{\rho(x, t)} \frac{\partial P(x, t)}{\partial x}=\rho(x, t) \frac{d p(\rho)}{d \rho} \frac{\partial V(x, t)}{\partial x} .
$$

Therefore, the traffic pressure according to the model of Aw and Rascle is a function of the velocity gradient rather than the density gradient, in contrast to Payne's pressure term (57). Consequently, Aw's and Rascle's pressure term must result in a different way than Payne's one, i.e. from a different kind of car-following model [18]. In order to illustrate this, let us now discuss a generalization of the optimal velocity model and its macroscopic counterpart.

\subsubsection{Non-local macroscopic traffic models}

It is well-known [24] that the optimal velocity model may produce accidents, if the initial condition, the optimal velocity function $v_{\mathrm{o}}(d)$, and the parameter $\tau$ are not carefully chosen. In order to have both, the emergence of traffic jams and the avoidance of accidents, we need to assume that the repulsive interaction force among vehicles does not only depend on the vehicle distance $d_{i}(t)=x_{i-1}(t)-x_{i}(t)$, but also on the vehicle velocity $v_{i}(t)$ (to reflect the dependence of the safe distance on the vehicle speed) or on the relative velocity

$$
\Delta v_{i}(t)=v_{i}(t)-v_{i-1}(t)=-\frac{d d_{i}}{d t}
$$

The corresponding generalization of the acceleration equation (11) reads

$$
\frac{d v_{i}}{d t}=a_{i}(t)=\frac{v^{0}-v_{i}(t)}{\tau}+f\left(d_{i}(t), v_{i}(t), \Delta v_{i}(t)\right) .
$$

This also changes the associated macroscopic traffic equation. Namely, equation (47) has to be replaced by

$$
\begin{aligned}
\frac{\partial V(x, t)}{\partial t}+ & V(x, t) \frac{\partial V(x, t)}{\partial x}= \\
- & \frac{1}{\rho(x, t)} \frac{\partial}{\partial x}[\rho(x, t) \theta(x, t)]+\frac{v^{0}-V(x, t)}{\tau} \\
& +\frac{1}{2} f\left(\frac{1}{\rho(x, t)}, V(x, t), \Delta V(x, t)\right) \\
+\frac{1}{2} f( & \left.\frac{1}{\rho(x+1 / \rho, t)}, V(x+1 / \rho, t), \Delta V(x+1 / \rho, t)\right) .
\end{aligned}
$$

For the sake of comparison with other macroscopic traffic models and linear stability analyses, let us perform a 
Taylor approximation of this. First, we may write

$$
\begin{aligned}
f\left(\frac{1}{\rho(x+1 / \rho, t)}, \Delta V(x+1 / \rho, t), V(x+1 / \rho, t)\right) \approx \\
f\left(\frac{1}{\rho(x, t)}, \Delta V(x, t), V(x, t)\right) \\
+\frac{\partial f}{\partial d} \frac{d d}{d \rho}[\rho(x+1 / \rho, t)-\rho(x, t)] \\
+\frac{\partial f}{\partial v}[V(x+1 / \rho, t)-V(x, t)] \\
+\frac{\partial f}{\partial \Delta v}[\Delta V(x+1 / \rho, t)-\Delta V(x, t)] .
\end{aligned}
$$

Then, we may insert $d d / d \rho=-1 / \rho^{2}$,

$$
\begin{aligned}
\rho(x+1 / \rho, t)-\rho(x, t) & \approx \frac{\partial \rho}{\partial x} \frac{1}{\rho}, \\
V(x+1 / \rho, t)-V(x, t) & \approx \frac{\partial V}{\partial x} \frac{1}{\rho} .
\end{aligned}
$$

Furthermore, considering $\Delta v_{i}(t)=-d d_{i} / d t, \rho(x, t)=$ $1 / d_{i}(t)$, and the continuity equation $d \rho / d t=\partial \rho / \partial t+$ $V \partial \rho / \partial x=-\rho \partial V / \partial x$, we get

$$
\begin{aligned}
\Delta V(x, t) & =-\frac{d}{d t}\left(\frac{1}{\rho(x, t)}\right)=\frac{1}{\rho(x, t)^{2}} \frac{d \rho(x, t)}{d t} \\
& =-\frac{1}{\rho(x, t)} \frac{\partial V(x, t)}{\partial x} \\
& \approx V(x, t)-V(x+1 / \rho, t)
\end{aligned}
$$

and

$$
\begin{aligned}
\Delta V(x+1 / \rho, t)-\Delta V(x, t) & \approx \frac{\partial \Delta V}{\partial x} \frac{1}{\rho} \\
& \approx-\frac{1}{\rho} \frac{\partial}{\partial x}\left(\frac{\partial V}{\partial x} \frac{1}{\rho}\right) \\
& =\frac{1}{\rho^{3}} \frac{\partial \rho}{\partial x} \frac{\partial V}{\partial x}-\frac{1}{\rho^{2}} \frac{\partial^{2} V}{\partial x^{2}} \\
& \approx-\frac{1}{\rho^{2}} \frac{\partial^{2} V}{\partial x^{2}},
\end{aligned}
$$

since a linearization drops products of gradient terms such as $(\partial \rho / \partial x)(\partial V / \partial x)$ (which are assumed to be smaller than the linear terms). Altogether, with $d d / d \rho=-1 / \rho^{2}$ we can write

$$
\begin{array}{r}
f\left(\frac{1}{\rho(x+1 / \rho, t)}, V(x+1 / \rho, t), \Delta V(x+1 / \rho, t)\right) \approx \\
f\left(\frac{1}{\rho(x, t)}, \Delta V(x, t), V(x, t)\right)-\frac{1}{\rho^{3}} \frac{\partial f}{\partial d} \frac{\partial \rho}{\partial x} \\
+\frac{1}{\rho} \frac{\partial f}{\partial v} \frac{\partial V}{\partial x}-\frac{1}{\rho^{2}} \frac{\partial f}{\partial v} \frac{\partial^{2} V}{\partial x^{2}} .
\end{array}
$$

With the definition

$$
V_{\mathrm{o}}(\rho, V, \Delta V)=v^{0}+\tau f\left(\frac{1}{\rho}, V, \Delta V\right),
$$

we may finally write

$$
\begin{array}{r}
\frac{\partial V(x, t)}{\partial t}+V(x, t) \frac{\partial V(x, t)}{\partial x}=-\frac{1}{\rho} \frac{\partial}{\partial x}[\rho(x, t) \theta(x, t)] \\
+\frac{V_{\mathrm{o}}(\rho, V, \Delta V)-V(x, t)}{\tau}-\frac{1}{2 \rho^{3}} \frac{\partial f}{\partial d} \frac{\partial \rho}{\partial x} \\
+\frac{1}{2 \rho} \frac{\partial f}{\partial v} \frac{\partial V}{\partial x}-\frac{1}{2 \rho^{2}} \frac{\partial f}{\partial v} \frac{\partial^{2} V}{\partial x^{2}} .
\end{array}
$$

Furthermore, let us assume that the variance can be approximated as a function of the density and the average velocity:

$$
\theta(x, t)=\theta_{\mathrm{e}}(\rho(x, t), V(x, t)) .
$$

With the definitions

$$
\begin{aligned}
\frac{\partial P_{1}}{\partial \rho} & =\theta_{\mathrm{e}}(\rho, V)+\rho \frac{\partial \theta_{\mathrm{e}}(\rho, V)}{\partial \rho}+\frac{1}{2 \rho^{2}} \frac{\partial f(1 / \rho, V, \Delta V)}{\partial d}, \\
\frac{\partial P_{2}}{\partial V} & =\rho \frac{\partial \theta_{\mathrm{e}}(\rho, V)}{\partial V}-\frac{1}{2} \frac{\partial f(1 / \rho, V, \Delta V)}{\partial v} \\
\eta & =-\frac{1}{2 \rho^{2}} \frac{\partial f(1 / \rho, V, \Delta V)}{\partial \Delta v}
\end{aligned}
$$

(where $\eta$ should be greater than zero), we may also write the linearized macroscopic traffic equations as

$$
\begin{aligned}
& \frac{\partial V(x, t)}{\partial t}+V(x, t) \frac{\partial V(x, t)}{\partial x}=-\frac{1}{\rho} \frac{\partial P_{1}}{\partial \rho} \frac{\partial \rho}{\partial x} \\
&-\frac{1}{\rho} \frac{\partial P_{2}}{\partial V} \frac{\partial V}{\partial x}+\eta \frac{\partial^{2} V}{\partial x^{2}} \\
&+\frac{V_{\mathrm{o}}(\rho, \Delta V, V)-V(x, t)}{\tau} .
\end{aligned}
$$

The term $\eta \partial^{2} V / \partial x^{2}$ can be interpreted as viscosity term and has a smoothing effect. Further viscosity (and diffusion) terms may be derived by second-order Taylor expansions. It is interesting to note that the pressure term containing $P_{2}$ looks similar to equation (61). Therefore, it is possible to derive Aw's and Rascle's model from a suitably specified microscopic traffic model [18].

\section{Summary, discussion, and conclusions}

In this paper, we have discussed several approaches to derive macroscopic traffic equations from microscopic carfollowing models. It has been pointed out that a Taylor approximation may be used only for linear stability analyses, as the gradients would otherwise often be too large for the approximation to work. Further undesireable consequences of a gradient expansion are the possible occurence of negative velocities, diffusion instabilities, and inefficient numerical solution methods.

The linear interpolation approach often works well in practise [17], but it is theoretically inconsistent as it violates the continuity equation which is required for the conservation of the vehicle number. In contrast, the approach reminding of smoothed particle hydrodynamics was suited 
in all respects. It led to a non-local macroscopic traffic model, which partially reminds of the non-local gas-kinetic based traffic model [9] (see Appendix A). In order to reach a realistic traffic dynamics (in particular accident avoidance if a vehicle with speed $v^{0}$ approaches a standing car), one needs to take into account that the repulsive vehicle interactions not only depend on the vehicle distance, but also on the relative velocity and the vehicle velocity. This leads to a specification of the traffic pressure which contains variance-dependent terms, additional terms proportional to $\partial \rho / \partial x$ as in Payne's model, and further terms proportional to $\partial V / \partial x$ as in Aw's and Rascle's model. While the variance-dependent term describes dispersion effects, Payne's, Aw's and Rascle's terms reflect effects of vehicle interactions. Furthermore note that, in case of multi-lane traffic, the additional inter-lane variance

$$
\Theta(x, t)=\frac{1}{L} \sum_{l=1}^{L} \frac{\rho_{l}(x, t)}{\rho(x, t)}\left[V_{l}(x, t)-V(x, t)\right]^{2},
$$

must be added to the inner-lane variance $\theta(x, t)$, where $\rho_{l}(x, t)$ is the density and $V_{l}(x, t)$ the average velocity in lane $l$ at location $x$ and time $t[2,25]$.

Let us finally discuss whether the above "smoothed particle hydrodynamics approach" may lead to inconstencies such as extremely high densities. An unrealistic car-following model may, in fact, imply a theoretically inconsistent macroscopic traffic model, but a plausible microscopic model should generate a plausible macroscopic one: specifically, the preservation of the order of vehicles requires a car-following model that does not produce accidents. Examples for this are the intelligent driver model (IDM) [26] or the Gipps model [27]. Furthermore, if the car-following model implies that vehicles keep a minimum distance of $d_{\mathrm{min}}$, as the IDM does, this will translate into a maximum density $\rho_{\text {jam }}=1 / d_{\text {min }}$ in the equivalent macroscopic traffic model. This can be seen from equation (43) with $\rho=1 / d_{\min }$. Therefore, in order to obtain a realistic macroscopic traffic model, one needs to make a suitable specification of the repulsive interaction force $f$. Generally, it is advised to work with speed-dependent interaction forces. An example for a microcopically derived macroscopic traffic model that takes into account the finite space requirements of vehicles is the non-local gas-kinetic-based traffic model (see Appendix A).

The author would like to thank for the inspiring discussions with the participants of the Workshop on "Multiscale Problems and Models in Traffic Flow" organized by Michel Rascle and Christian Schmeiser at the Wolfgang Pauli Institute in Vienna from May 5-9, 2008, with partial support by the CNRS.

\section{Appendix A: The non-local, gas-kinetic based traffic model}

For comparison, let us shortly recall the form of the nonlocal gas-kinetic based traffic model (GKT model). This has been derived via a collision approximation [9] and can be written in the form of equation (56) with $P(x, t)=$ $\rho(x, t) \theta(x, t)$, but $V_{\mathrm{e}}(\rho)$ must be replaced by a non-local expression

$$
V_{\mathrm{g}}\left(\rho, V, \theta, \rho_{+}, V_{+}, \theta_{+}\right)=v^{0} \underbrace{-\tau\left[1-p\left(\rho_{+}\right)\right] \chi\left(\rho_{+}\right) \rho_{+} B(\Delta)}_{\text {repulsive interaction term }} .
$$

Here, the index "+" indicates evaluation at the advanced "interaction point" $x+s_{0}+T V$, where $s_{0}$ represents the minimum vehicle distance and $T V$ the velocity-dependent safety distance. The related non-locality has some effects that other macroscopic models generate by their pressure and viscosity terms. The dependence of the non-local repulsive interaction on the effective dimensionless velocity difference

$$
\Delta=\frac{V-V_{+}}{\sqrt{\theta-2 r \sqrt{\theta \theta_{+}}+\theta_{+}}}
$$

takes into account effects of the velocity variances $\theta, \theta_{+}$, and velocity correlations $r$ among successive cars [25]. Furthermore, the "Boltzmann factor"

$$
B(\Delta)=\left(\theta-2 r \sqrt{\theta \theta_{+}}+\theta_{+}\right)\left[\Delta N(\Delta)+\left(1+\Delta^{2}\right) E(\Delta)\right]
$$

in the braking term is monotonically increasing with $\Delta V$. It contains the normal distribution

$$
N(\Delta)=\frac{\mathrm{e}^{-\Delta^{2} / 2}}{\sqrt{2 \pi}}
$$

and the Gaussian error function

$$
E(\Delta)=\int_{-\infty}^{\Delta} d z N(z)
$$

To close the system of equations, the velocity correlation $r$ is specified as a function of the density in accordance with empirical observations. Moreover, for a description of the presently known properties of traffic flows it seems sufficient to set

$$
\theta=A(\rho) V^{2}
$$

This guarantees that the velocity variance will vanish whenever the average velocity goes to zero, but it will be positive otherwise. It should be noted that the variance prefactor $A$ is higher in congested traffic than in free traffic [9]. The "effective cross section" is, finally, specified via

$$
[1-p(\rho)] \chi(\rho)=\frac{v^{0} \rho T^{2}}{\tau A\left(\rho_{\mathrm{jam}}\right)\left(1-\rho / \rho_{\mathrm{jam}}\right)^{2}},
$$

where $T$ is the safe time headway and $\rho_{\text {jam }}$ the maximum vehicle density. This formula makes also sense in the lowdensity limit $\rho \rightarrow 0$, where $\chi \rightarrow 1$ and $p \rightarrow 1$.

A linear stability analysis of the non-local traffic model can be done via a gradient expansion. It results in equations of the kind (77) and further viscosity and diffusion terms [28]. 


\section{References}

1. D. Chowdhury, L. Santen, A. Schadschneider, Physics Reports 329, 199 (2000)

2. D. Helbing, Rev. Modern Phys. 73, 1067 (2001)

3. T. Nagatani, Reports on Progress in Physics 65, 1331 (2002)

4. K. Nagel, Multi-Agent Transportation Simulations, see http://www2.tu-berlin.de/fb10/ISS/FG4/archive/ sim-archive/publications/book/

5. R.D. Kühne, M.B. Rödiger, in Proceedings of the 1991 Winter Simulation Conference, edited by B.L. Nelson, W.D. Kelton, G.M. Clark (Society for Computer Simulation International, Phoenix, AZ, 1991), pp. 762-770

6. M. Papageourgiou, Applications of Automatic Control Concepts to Traffic Flow Modeling and Control (Springer, Heidelberg, 1983)

7. B.S. Kerner, P. Konhäuser, Phys. Rev. E 48, 2335 (1993)

8. D. Helbing, M. Treiber, Granular Matter 1, 21 (1998)

9. M. Treiber, A. Hennecke, D. Helbing, Phys. Rev. E 59, 239 (1999)

10. H.J. Payne, in Mathematical Models of Public Systems, edited by G.A. Bekey (Simulation Council, La Jolla, CA, 1971), Vol. 1, pp. 51-61

11. H.J. Payne, in Research Directions in Computer Control of Urban Traffic Systems, edited by W.S. Levine, E. Lieberman, J.J. Fearnsides (American Society of Civil Engineers, New York, 1979), pp. 251-265.
12. G.B. Whitham, Linear and Nonlinear Waves (Wiley, New York, 1974)

13. G.F. Newell, Operations Research 9, 209 (1961)

14. M. Bando, K. Hasebe, A. Nakayama, A. Shibata, Y. Sugiyama, Phys. Rev. E 51, 1035 (1995)

15. P. Berg, A. Mason, A. Woods, Phys. Rev. E 61, 1056 (2000)

16. H.K. Lee, H.-W. Lee, D. Kim, Phys. Rev. E 64, 056126 (2001)

17. D. Helbing, A. Hennecke, V. Shvetsov, M. Treiber, Mathematical and Computer Modelling 35, 517 (2002)

18. A. Aw, A. Klar, T. Materne, M. Rascle, SIAM J. Appl. Math. 63, 259 (2002)

19. S. Moutari, M. Rascle, SIAM J. Appl. Math. 68, 413 (2007)

20. D. Helbing, M. Treiber, Computing in Science \& Engineering 1, 89 (1999)

21. C.F. Daganzo, Transportation Research B 29, 277 (1995)

22. D. Helbing, Eur. Phys. J. B (2009),

DOI : $10.1140 /$ epjb/e2009-00182-7

23. A. Aw, M. Rascle, SIAM Journal on Applied Mathematics 60, 916 (2000)

24. D. Helbing, M. Schreckenberg, Phys. Rev. E 59, R2505 (1999)

25. V. Shvetsov, D. Helbing, Phys. Rev. E 59, 6328 (1999)

26. M. Treiber, A. Hennecke, D. Helbing, Phys. Rev. E 62 , 1805 (2000)

27. P.G. Gipps, Transpn. Res. B 15, 105 (1981)

28. D. Helbing, Physica A 233, 253 (1996), see also http:// arxiv.org/abs/cond-mat/9805136 